Vietnam Journal of Mechanics, VAST, Vol.40, No. 4 (2018), pp. 303 -323

DOI: https://doi.org/10.15625/0866-7136/13497

\title{
EFFECTIVE BOUNDARY CONDITION FOR THE REFLECTION OF SHEAR WAVES AT THE PERIODIC ROUGH BOUNDARY OF AN ELASTIC BODY
}

\author{
Agnès Maurel $^{1}$, Jean-Jacques Marigo ${ }^{2, *}$, Kim Pham $^{3}$ \\ ${ }^{1}$ Institut Langevin, CNRS, ESPCI ParisTech, France \\ ${ }^{2}$ Laboratoire de Mécanique du Solide, CNRS, Ecole Polytechnique, France \\ ${ }^{3}$ Unité de Mécanique, ENSTA ParisTech, France \\ *E-mail: marigo@lms.polytechnique.fr \\ Received Frebuary 07, 2018
}

\begin{abstract}
We present a homogenization method to treat the problem of the reflection of waves at the free boundary of an elastic body, the edge being structured periodically at the subwavelength scale. The problem is considered for shear waves and the wave equation in the time domain is considered. In the homogenized problem, a boundary condition at an equivalent flat edge is obtained, which links the normal stress to its derivatives, instead of the usual traction free condition. The problem of the position of the equivalent flat boundary with respect to the real roughnesses is addressed and this is done considering the equation of energy conservation in the homogenized problem and considering the accuracy of the homogenized solution when compared to the real one.
\end{abstract}

Keywords: homogenization method, reflection of waves, rough free boundary, subwavelength scale, shear waves, energy conservation.

\section{INTRODUCTION}

The problem of the propagation of waves in complex geometries most often cannot be solved analytically and it requires a numerical resolution. Among the different source of complexity, the existence of very different length scales is the worth in terms of numerical computations, at least when one expects that both the smallest and the largest scales have an impact on the wave behavior; this is because the mesh has to resolve the rapid variations associated to the small scale while the computational domain has be sized with the largest scale. If the smallest scale is the scale of the typical wavelength, which means the high frequency regime, there is no miracle and the numerics will be demanding. In the opposite case, typically for subwavelength heterogeneities of the medium, homogenization approaches are well adapted to simplify considerably the problem; owing to the definition of a small parameter being the ratio of the small scale to the large scale, it is possible to derive of an equivalent homogenized problem, in which the small scale

(C) 2018 Vietnam Academy of Science and Technology 
has disappeared. This can happen when the whole propagation medium (or a large part) is microstructured in which case classical homogenization offers an equivalent homogeneous (and possibly anisotropic) medium, see e.g. [1]. In other configurations, only a small, or say a thin, region contains a microstructuration; originally developed in the context of solid mechanics [2,3], interface homogenizations have been applied to such configurations mainly in acoustics [4,5] and in electromagnetism [6-9].

We consider such configuration, namely the reflection of shear waves by the rough free boundary of an elastic body. The roughnesses are periodic and satisfy $\varepsilon=k h, k e \ll 1$ with $k$ the typical wavenumber and $(h, e)$ the size of the roughness (Fig. 1), which corresponds typically to the Rayleigh criterion for rough surfaces [10]. We show that this problem can be replaced by an equivalent one in which an effective boundary condition on a flat boundary replaces the usual condition of vanishing normal stress, see (23); this is done in Section 2. In fact, the homogenized problem is not unique since the equivalent boundary can be placed at different positions in the elastic body. Although these homogenized problems are equivalent up to the $O\left(\varepsilon^{2}\right)$, all are not acceptable when the equation of energy conservation is considered; besides, they do not yield the same accuracy when a particular scattering problem is considered. We address these two aspects in Section 3. We collect in the appendices the technical calculations used to solve (i) the elementary problem yielding the parameter entering in the effective boundary condition and (ii) the simple scattering problem considered in Section 3.1; the corresponding scripts are provided.
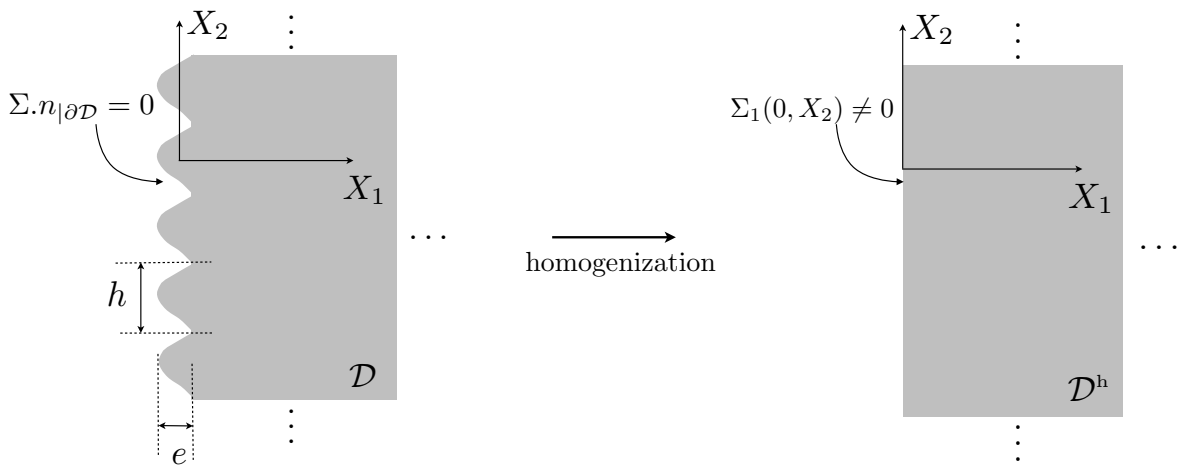

Fig. 1. On the left, the actual configuration of an elastic body (in grey) with a free rough boundary. On the right, the homogenized configuration where the rough boundary is replaced by a flat one where the boundary condition is non classical

\section{HOMOGENIZATION OF THE ROUGH BOUNDARY}

\subsection{Position of the problem and notations}

We start with the Navier equations written in terms of $V(\mathbf{X}, t)=\partial_{t} U(\mathbf{X}, t)$ being the time derivative of the scalar displacement $U(\mathbf{X}, t)$ (and $U$ is anti-plane) and of the stress vector $\boldsymbol{\Sigma}(\mathbf{X}, t)$, written in the time domain, with $t$ the time and $\mathbf{X}=\left(X_{1}, X_{2}\right)$ the spatial 
coordinates

$$
\left\{\begin{array}{l}
\frac{\partial \Sigma}{\partial t}=\mu \nabla V \\
\rho \frac{\partial V}{\partial t}=\operatorname{div} \Sigma
\end{array}\right.
$$

with $\rho$ and $\mu$ being the mass density and the shear modulus respectively. From (1), we can deduce the equation of energy conservation in a bounded domain $\mathcal{D}$

$$
\frac{d}{d t} \mathcal{E}+\int_{\partial \mathcal{D}} d S \Pi \cdot \mathbf{n}=0
$$

with $\varepsilon$ the usual elastic energy and $\Pi$ the Poynting vector

$$
\mathcal{E}=\int_{\mathcal{D}} d V\left[\frac{\rho}{2} V^{2}+\frac{1}{2 \mu}|\Sigma|^{2}\right], \quad \Pi=V \Sigma
$$

In the time domain, we consider elastic waves with a minimum wavelength $2 \pi / k$ larger than the typical periodicity of the roughnesses $h$, such that

$$
\varepsilon \equiv k h \ll 1 \text {. }
$$

To be consistent, we shall work in dimensionless coordinate, and to do that, we define

$$
\mathbf{x} \equiv k \mathbf{X}, \quad \tau \equiv k c t
$$

and

$$
\sigma^{\varepsilon}(\mathbf{x}, \tau) \equiv \frac{c}{\mu} \Sigma(\mathbf{X}, t), \quad v^{\varepsilon}(\mathbf{x}, \tau) \equiv V(\mathbf{X}, t),
$$

where $c=\sqrt{\mu / \rho}$ is the celerity of the shear waves in the elastic body. Also, we indicated explicitly the dependence of $\left(\sigma^{\varepsilon}, v^{\varepsilon}\right)$ on $\varepsilon$ being the periodicity of the roughnesses in non dimensional form. Now, (1) read

$$
\left\{\begin{array}{l}
\frac{\partial \sigma^{\varepsilon}}{\partial \tau}=\nabla_{\mathbf{x}} v^{\varepsilon} \\
\frac{\partial v^{\varepsilon}}{\partial \tau}=\operatorname{div}_{\mathbf{x}} \sigma^{\varepsilon}
\end{array}\right.
$$

\subsection{The matched asymptotic expansion}

\subsubsection{Inner and outer expansions}

The idea is to expand the solutions of (3) with respect to the small parameter $\varepsilon$, namely

$$
\left\{\begin{array}{l}
v^{\varepsilon}=v^{0}(\mathbf{x}, \tau)+\varepsilon v^{1}(\mathbf{x}, \tau)+\varepsilon^{2} v^{2}(\mathbf{x}, \tau)+\ldots \\
\sigma^{\varepsilon}=\sigma^{0}(\mathbf{x}, \tau)+\varepsilon \sigma^{1}(\mathbf{x}, \tau)+\varepsilon^{2} \sigma^{2}(\mathbf{x}, \tau)+\ldots
\end{array}\right.
$$

In principle, this expansion can be used in the whole space (see e.g. [11]). Nevertheless, if the spatial derivatives in Eq. (3) make $\varepsilon$ to appear, the resolution may become tricky. Such complications are avoided if a displacement in $\mathbf{x}$ of order unity produces a variation in $v^{\varepsilon}$ and $\sigma^{\varepsilon}$ of order unity, namely $\partial_{x_{i}} v^{\varepsilon} \sim v^{\varepsilon}$. This is ensured in the far field, that is far enough from the rough boudary, where $\partial_{X_{i}} V \sim k V \rightarrow \partial_{x_{i}} v^{\varepsilon} \sim v^{\varepsilon}$. The story is different in near field: there, the roughnesses generate evanescent waves 
whose strongest variations are associated to the smallest scales of the roughnesses, say $\partial_{X_{i}} V \sim V / h \rightarrow \partial_{x_{i}} v^{\varepsilon} \sim v^{\varepsilon} / \varepsilon$. Also, the wavefield has in general variations when moving along the free boundary which are associated to the typical central wavelength. This behavior is associated to slow variations of $v^{\varepsilon}$ (for which $\partial_{x_{2}} v^{\varepsilon} \sim v^{\varepsilon}$ ). Thus, we have to deal with different scales in the far and near fields, and with two scales in the near field.

The presented approach solves these problems with two ingredients: first, a separation of the space into an inner and an outer regions, which correspond to the near and far fields, respectively. In the outer region, the natural coordinates $\mathbf{x} \equiv\left(x_{1}, x_{2}\right)$ are adapted and the expansion in (4) applies. In the inner region, the rapid variations of $v^{\varepsilon}$ and $\sigma^{\varepsilon}$ are accounted introducing a new system of coordinates $\mathbf{y}=\mathbf{x} / \varepsilon$ such that $\partial_{y_{i}} v^{\varepsilon} \sim v^{\varepsilon}$ accounts for the rapid variations of the evanescent field of $v^{\varepsilon}$; next, the slow variations along $x_{2}$ are accounted for by keeping $x_{2}$ as additional coordinate. Note that in the $y$ coordinates, the cell has a periodicity equal to unity (Fig. 2). Owing to this modification, the expansions read

$$
\begin{aligned}
& \text { Outer exp. }\left\{\begin{array}{l}
v^{\varepsilon}=v^{0}(\mathbf{x}, \tau)+\varepsilon v^{1}(\mathbf{x}, \tau)+\ldots, \\
\sigma^{\varepsilon}=\sigma^{0}(\mathbf{x}, \tau)+\varepsilon \sigma^{1}(\mathbf{x}, \tau)+\ldots
\end{array}\right. \\
& \text { Inner exp. }\left\{\begin{array}{l}
v^{\varepsilon}=w^{0}\left(\mathbf{y}, x_{2}, \tau\right)+\varepsilon w^{1}\left(\mathbf{y}, x_{2}, \tau\right)+\ldots \\
\sigma^{\varepsilon}=\tau^{0}\left(\mathbf{y}, x_{2}, \tau\right)+\varepsilon \tau^{1}\left(\mathbf{y}, x_{2}, \tau\right)+\ldots
\end{array}\right.
\end{aligned}
$$

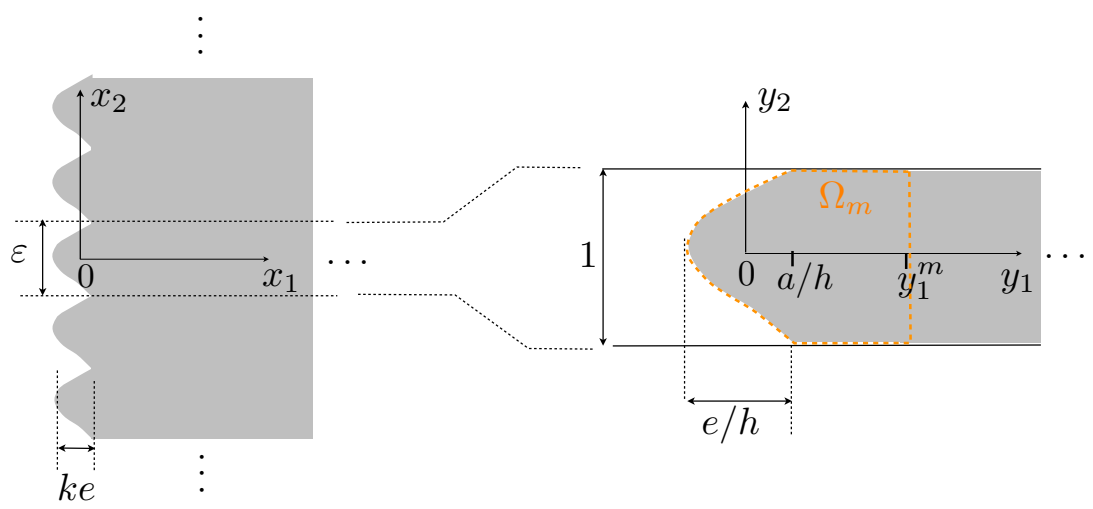

Fig. 2. The elastic body with rough free boundary in the two systems of coordinates $\mathbf{x}$ and $\mathbf{y}$. In $\mathbf{y}$-coordinates, $\mathcal{S}=e \varphi / h$ denotes the surface of air in the roughnesses in dimensionless form 
Now, (3) can be written in the inner and in the outer regions, owing to the expressions of the differential operator

$$
\begin{cases}\nabla \rightarrow \nabla_{x}, & \text { in the outer problem, } \\ \nabla \rightarrow \frac{1}{\varepsilon} \nabla_{y}+\frac{\partial}{\partial x_{2}} \mathbf{e}_{2}, & \text { in the inner problem. }\end{cases}
$$

\subsubsection{Equations at orders 0 and 1}

We start by reporting the equations that will be needed in the following. For the outer solution, using (6a), Eqs. (3) become for $n=0$ and 1

$$
\left\{\begin{array}{l}
\frac{\partial \sigma^{n}}{\partial \tau}=\nabla_{\mathbf{x}} v^{n} \\
\frac{\partial v^{n}}{\partial \tau}=\operatorname{div}_{\mathbf{x}} \sigma^{n}
\end{array}\right.
$$

and for the inner solution, using (6b), we get

$$
\left\{\begin{array}{l}
\nabla_{\mathbf{y}} w^{0}=0 \\
\operatorname{div}_{\mathbf{y}} \boldsymbol{\tau}^{0}=\mathbf{0} \\
\frac{\partial \tau^{0}}{\partial \tau}=\frac{\partial w^{0}}{\partial x_{2}} \mathbf{e}_{2}+\nabla_{\mathbf{y}} w^{1} \\
\frac{\partial w^{0}}{\partial \tau}=\frac{\partial \tau_{2}^{0}}{\partial x_{2}}+\operatorname{div}_{\mathbf{y}} \boldsymbol{\tau}^{1}
\end{array}\right.
$$

Next, (7)-(8) together with the boundary conditions and the matching conditions will be used to find the conditions to be applied on an equivalent surface.

\subsubsection{Boundary conditions and matching conditions}

Because of the separation of the space into two regions, something has to be said on the boundary conditions. By construction, the stress- free boundary $\Gamma$ is seen by the inner solution only, whence

$$
\boldsymbol{\tau}^{n} \cdot \mathbf{n}_{\mid \Gamma}=0, n=0,1, \ldots
$$

However, the condition to be applied for $y_{1} \rightarrow+\infty$ is unknown a priori. Reversely, the condition to be applied to the outer solution for $x_{1} \rightarrow+\infty$ is known as soon as the source has been defined (this condition is in general a condition of the Sommerfield type, also called radiation condition). However, the outer solution does not see the boundary and thus, the condition for $x_{1} \rightarrow 0$ is unknown; it is in fact the condition we are looking for. These missing conditions for the inner and outer solutions are provided by so-called matching conditions. Basically, the idea is that the two solutions have to match in some intermediate region. They are written following [2] for $x_{1} \rightarrow 0^{+}$ corresponding to $y_{1} \rightarrow+\infty$. To do so, we use Taylor expansions of $v^{0}\left(x_{1}, x_{2}, \tau\right)=$ $v^{0}\left(0^{+}, x_{2}, \tau\right)+x_{1} \partial_{x_{1}} v^{0}\left(0^{+}, x_{2}, \tau\right)+\cdots=v^{0}\left(0^{+}, x_{2}, \tau\right)+\varepsilon y_{1} \partial_{x_{1}} v^{0}\left(0^{+}, x_{2}, \tau\right)+\ldots$, same for $\sigma^{0}$. Identifying the terms in $\varepsilon^{n}, n=0,1$ in the inner and outer expansions, Eqs. (5), 
we get, for $n=0$

$$
\left\{\begin{array}{l}
v^{0}\left(0^{+}, x_{2}, \tau\right)=\lim _{y_{1} \rightarrow+\infty} w^{0}\left(\mathbf{y}, x_{2}, \tau\right), \\
\sigma^{0}\left(0^{+}, x_{2}, \tau\right)=\lim _{y_{1} \rightarrow+\infty} \tau^{0}\left(\mathbf{y}, x_{2}, \tau\right),
\end{array}\right.
$$

and for $n=1$, we shall need only the matching condition for $\sigma^{1}$

$$
\sigma^{1}\left(0^{+}, x_{2}, \tau\right)=\lim _{y_{1} \rightarrow+\infty}\left[\tau^{1}\left(\mathbf{y}, x_{2}, \tau\right)-y_{1} \frac{\partial \sigma^{0}}{\partial x_{1}}\left(0^{+}, x_{2}, \tau\right)\right] .
$$

In the following, we use $\sigma^{n}\left(0, x_{2}, \tau\right)$, identifying the value of the function at $x_{1}=0$ to its limiting value. Also, when not needed, the dependancies of the functions on the spatial variables and on the time are omitted for readability.

\subsection{Determination of the equivalent boundary condition}

2.3.1. Solutions at order 0

First, (8a) tells us that $w_{0}$ does not depend on $y$, and from the matching condition (10a), we get

$$
w^{0}\left(x_{2}, \tau\right)=v^{0}\left(0, x_{2}, \tau\right)
$$

Next, integrating (8b) over $\Omega_{m}$ gives

$$
0=\int_{\Omega_{m}} \mathrm{~d} \mathbf{y} \operatorname{div}_{\mathbf{y}} \boldsymbol{\tau}^{0}=\int \mathrm{d} y_{2} \tau_{1}^{0}\left(y_{1}^{m}, y_{2}, x_{2}, \tau\right),
$$

where we have used the boundary condition (9) for $n=0$ on $\Gamma$ and the periodicity of $\tau^{0}$ w.r.t. $y_{2}$. Taking the limit $y_{1}^{m} \rightarrow+\infty$ along with the matching condition (10a), we get

$$
\sigma_{1}^{0}\left(0, x_{2}, \tau\right)=0 \text {. }
$$

At leading order, the rough boundary of the body behaves as a flat free boundary and we have to go to order 1 to capture the effect of the roughnesses.

\subsubsection{Solutions at order 1 and elementary problems}

Before going further, we need to define an elementary problem, which will make a surface parameter to appear. To that aim, we inspect (8b)-(8c), (9) and (10b) owing to (12); we get

$$
\left\{\begin{array}{l}
\Delta_{y} w^{1}=0, \\
{\left[\frac{\partial v^{0}}{\partial x_{2}}\left(0, x_{2}, \tau\right) \mathbf{e}_{2}+\nabla_{\mathbf{y}} w^{1}\right] \cdot \mathbf{n}_{\mid \Gamma}=0} \\
\lim _{y_{1} \rightarrow+\infty} \nabla_{\mathbf{y}} w^{1}=\frac{\partial v^{0}}{\partial x_{1}}\left(0, x_{2}, \tau\right) \mathbf{e}_{1}, \\
w^{1} \text { periodic } w . r . t . y_{2} .
\end{array}\right.
$$

The first and second equations are simply the time derivative versions of $(8 b)$ and of the boundary condition (9) for $n=0$, owing to (8c) in both cases. The third equation is less 
immediate although straightforward; consider the time derivative of (10b), with (8c)

$$
\frac{\partial \sigma^{0}}{\partial \tau}\left(0, x_{2}, \tau\right)=\lim _{y_{1} \rightarrow+\infty}\left[\frac{\partial v^{0}}{\partial x_{2}}\left(0, x_{2}, \tau\right) \mathbf{e}_{2}+\nabla_{y} w^{1}\right]
$$

which leads to the desired equation using (7a) for $n=0$. Now, because $\sigma_{1}^{0}\left(0, x_{2}, \tau\right)=0$, its time derivative is zero as well and from (7a), we have

$$
\frac{\partial \sigma_{1}^{0}}{\partial \tau}\left(0, x_{2}, \tau\right)=\frac{\partial v^{0}}{\partial x_{1}}\left(0, x_{2}, \tau\right)=0
$$

The system (14) reduces to

$$
\left\{\begin{array}{l}
\Delta_{y} w^{1}=0, \\
{\left[\frac{\partial v^{0}}{\partial x_{2}}\left(0, x_{2}, \tau\right) \mathbf{e}_{2}+\nabla_{\mathbf{y}} w^{1}\right] \cdot \mathbf{n}_{\mid \Gamma}=0} \\
\lim _{y_{1} \rightarrow+\infty} \nabla_{\mathbf{y}} w^{1}=\mathbf{0}, \\
w^{1} \text { periodic } w . r . t . y_{2}
\end{array}\right.
$$

being linear with respect to $\partial_{x_{2}} v^{0}\left(0, x_{2}, \tau\right)$. Thus, defining $W(\mathbf{y})$ such as

$$
w^{1}\left(\mathbf{y}, x_{2}, \tau\right)=\frac{\partial v^{0}}{\partial x_{2}}\left(0, x_{2}, \tau\right) W(\mathbf{y})+\tilde{w}\left(x_{2}, \tau\right)
$$

it is sufficient that $\mathrm{W}(\mathrm{y})$ satisfies

$$
\left\{\begin{array}{l}
\Delta W=0, \quad \text { in } \Omega, \\
{\left[\mathbf{e}_{2}+\nabla W\right] \cdot \mathbf{n}_{\mid \Gamma}=0,} \\
\lim _{y_{1} \rightarrow+\infty} \nabla W=\mathbf{0}, \\
W \text { periodic w.r.t. } y_{2},
\end{array}\right.
$$

to ensure that $w^{1}\left(\mathbf{y}, x_{2}, \tau\right)$ satisfies (15). The field $W$ is an evanescent field being excited on $\Gamma$ (and because $W$ is defined up to a constant, we can set this constant to 0 without loss of generality). Finally, note that $\tilde{w}\left(x_{2}, \tau\right)$ is introduced since (15) define $w_{1}$ up to a function independent of $y$; we shall see that the determination of $\tilde{w}\left(x_{2}, \tau\right)$ is not needed.

\subsubsection{Boundary condition at the equivalent surface and determination of the surface parameters}

To define the boundary condition, we want $\sigma_{1}^{1}\left(0, x_{2}, \tau\right)$ (we already know that $\sigma_{1}^{0}\left(0, x_{2}, \tau\right)=0$, and $\sigma_{1}^{1}\left(0, x_{2}, \tau\right)$ is given by $\tau_{1}^{1}\left(+\infty, y_{2}, x_{2}, \tau\right)$ in the matching condition (11). The limit of $\tau_{1}^{1}$ will be obtained by integrating (8d) over $\Omega_{m}$. But before, we inspect $\tau^{0}$ in (8c) (because $\tau_{2}^{0}$ is also needed in (8d)). Using (16) in (8c), we have

$$
\frac{\partial \tau^{0}}{\partial \tau}=\frac{\partial v^{0}}{\partial x_{2}}\left(0, x_{2}, \tau\right)\left[\mathbf{e}_{2}+\nabla_{\mathbf{y}} W\right]=\frac{\partial \sigma_{2}^{0}}{\partial \tau}\left(0, x_{2}, \tau\right)\left[\mathbf{e}_{2}+\nabla_{\mathbf{y}} W\right]
$$

and the latter equality is obtained using (7a). Assuming $\sigma^{0}=0$ and $\tau^{0}=0$ at $\tau=-\infty$ (the acoustic source has been switched on at some initial time), the above identity is valid 
omitting the time average, specifically

$$
\boldsymbol{\tau}^{0}=\sigma_{2}^{0}\left(0, x_{2}, \tau\right)\left[\mathbf{e}_{2}+\nabla_{\mathbf{y}} W\right] .
$$

We can come back to (8d), which is written

$$
\begin{aligned}
0 & =\frac{\partial v_{0}}{\partial \tau}\left(0, x_{2}, \tau\right)-\frac{\partial \sigma_{2}^{0}}{\partial x_{2}}\left(0, x_{2}, \tau\right)\left[1+\frac{\partial W}{\partial y_{2}}\right]-\operatorname{div}_{\mathbf{y}} \tau^{1} \\
& =-\frac{\partial \sigma_{2}^{0}}{\partial x_{2}}\left(0, x_{2}, \tau\right) \frac{\partial W}{\partial y_{2}}+\frac{\partial \sigma_{1}^{0}}{\partial x_{1}}\left(0, x_{2}, \tau\right)-\operatorname{div}_{\mathbf{y}} \boldsymbol{\tau}^{1}
\end{aligned}
$$

and the latter equality has been obtained using $(7 \mathrm{~b})$. It is now sufficient to integrate the above equation over $\Omega_{m}$ to find

$$
\frac{\partial \sigma_{2}^{0}}{\partial x_{2}}\left(0, x_{2}, \tau\right) \int_{\Omega_{m}} \mathrm{~d} \mathbf{y} \frac{\partial W}{\partial y_{2}}+\int \mathrm{d} y_{2}\left[\tau_{1}^{1}\left(y_{1}^{m}\right)-\left(y_{1}^{m}-\frac{a}{h}+\mathcal{S}\right) \frac{\partial \sigma_{1}^{0}}{\partial x_{1}}\left(0, x_{2}, \tau\right)\right]=0
$$

with $\mathcal{S}=\varphi e / h$ is the surface of the roughnesses in $\mathbf{y}$-coordinate (Fig. 3). Here, it is important to stress that $\left(y_{1}^{m}-a / h+\mathcal{S}\right)$ is the surface of the integration domain $\Omega_{m}$. It is independent on the choice of the origine $y_{1}=0$, but each term $y_{1}^{m}$ and $(\mathcal{S}-a / h)$ does depend on the origine $y_{1}=0$.

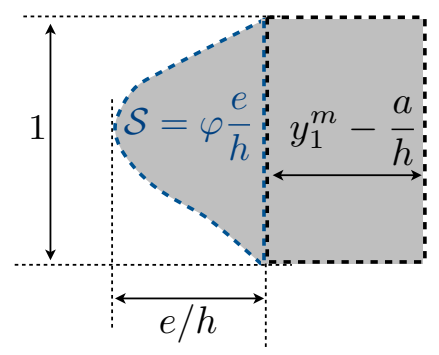

Fig. 3. Domain of integration $\Omega_{m}$ of surface $y_{1}^{m}-a / h+\mathcal{S}$; while this surface is independent of the choice of the origine in Fig. 2, both $y_{1}^{m}$ and $(\mathcal{S}-a / h)$ do depend on this choice

Taking the limit $y_{1}^{m} \rightarrow+\infty$ in the above equation, and using the matching condition (11), we finally get

$$
\sigma_{1}^{1}\left(0, x_{2}, \tau\right)=\left(\mathcal{S}-\frac{a}{h}\right) \frac{\partial \sigma_{1}^{0}}{\partial x_{1}}\left(0, x_{2}, \tau\right)+\mathcal{C} \frac{\partial \sigma_{2}^{0}}{\partial x_{2}}\left(0, x_{2}, \tau\right),
$$

where we have defined (with $\Omega=\lim _{y_{1}^{m} \rightarrow+\infty} \Omega_{m}$ )

$$
\mathcal{C} \equiv-\int_{\Omega} \mathrm{d} \mathbf{y} \frac{\partial W}{\partial y_{2}}
$$




\subsection{The final homogenized problem}

The equations in the bulk (7) and the associated boundary conditions (13) and (20) could be used to solve the homogenized problem iteratively: first compute $\left(v^{0}, \sigma^{0}\right)$ for a flat stress-free boundary (compute also $\mathcal{C}$ in (21)) and use the results to get the right handside term in (20); then, compute $\left(v^{1}, \sigma^{1}\right)$; finally, recompose $v^{0}+\varepsilon v^{1}$ which approximate $v^{\varepsilon}$ up to $O\left(\varepsilon^{2}\right)$. As discussed in [3], it is preferable to handle a unique problem and this is done by defining the fields $\left(v^{\mathrm{h}}, \sigma^{\mathrm{h}}\right)$ satisfying the following homogenized problem

$$
\left\{\begin{array}{l}
\frac{\partial \sigma^{\mathrm{h}}}{\partial \tau}=\nabla_{\mathbf{x}} v^{\mathrm{h}}, \quad \frac{\partial v^{\mathrm{h}}}{\partial \tau}=\operatorname{div}_{\mathbf{x}} \sigma^{\mathrm{h}}, \quad \text { for } x_{1}>0 \\
\sigma_{1}^{\mathrm{h}}\left(0, x_{2}, \tau\right)=\varepsilon\left(\mathcal{S}-\frac{a}{h}\right) \frac{\partial \sigma_{1}^{\mathrm{h}}}{\partial x_{1}}\left(0, x_{2}, \tau\right)+\varepsilon \mathcal{C} \frac{\partial \sigma_{2}^{\mathrm{h}}}{\partial x_{2}}\left(0, x_{2}, \tau\right) .
\end{array}\right.
$$

A quick analysis of (7), (13) and (20) shows that $v^{\mathrm{h}}$ has the same expansion as $v^{0}+v^{1}$, and thus as $v^{\varepsilon}$, up to $O\left(\varepsilon^{2}\right)$ (the same for $\sigma^{\mathrm{h}}$ ). Finally, coming back to the real space (through (2)), we get

$$
\left\{\begin{array}{l}
\frac{\partial \Sigma^{\mathrm{h}}}{\partial t}=\mu \nabla V^{\mathrm{h}}, \quad \rho \frac{\partial V^{\mathrm{h}}}{\partial t}=\operatorname{div} \Sigma^{\mathrm{h}}, \quad \text { in } \mathcal{D}^{\mathrm{h}}, \\
\Sigma_{1}^{\mathrm{h}}\left(0, X_{2}, t\right)=(e \varphi-a) \frac{\partial \Sigma_{1}^{\mathrm{h}}}{\partial X_{1}}\left(0, X_{2}, t\right)+h \mathcal{C} \frac{\partial \Sigma_{2}^{\mathrm{h}}}{\partial X_{2}}\left(0, X_{2}, t\right), \\
\text { the appropriate radiation condition at } X_{1} \rightarrow+\infty .
\end{array}\right.
$$

The above homogenized problem depends on the choice of a because of the boundary condition (in an obvious manner; note that $\mathcal{C}$ does not depend on $a$ ) but also because of the domain $\mathcal{D}^{h}$ (Fig. 1). In the following section, we address the problem of the choice of $a$.

\section{DEPENDANCE OF THE HOMOGENIZED PROBLEM ON THE $\alpha$-VALUE}

\subsection{Energy conservation in the homogenized problem}

\subsubsection{The boundary energy in the equation of energy conservation}

The choice of the origine $y_{1}=0$ affects the boundary condition in (23) and the position of the equivalent boundary (notably it is within the actual roughnesses for $-e<a<$ e). In the original problem, the elastic energy is

$$
\mathcal{E}=\int_{\mathcal{D}} \mathrm{d} V\left[\frac{\rho}{2} V^{2}+\frac{1}{2 \mu}|\Sigma|^{2}\right]
$$

and in the homogenized problem, it has to be of form

$$
\mathcal{E}_{\mathrm{h}}=\int_{\mathcal{D}_{h}} \mathrm{~d} V\left[\frac{\rho}{2} V^{\mathrm{h} 2}+\frac{1}{2 \mu}\left|\Sigma^{\mathrm{h}}\right|^{2}\right]+\mathcal{E}_{b}
$$

where $\mathcal{E}_{b}$ is the energy of the equivalent boundary. In the absence of losses, the equations of energy conservation read $d \mathcal{E} / d t=0=d \mathcal{E}_{\mathrm{h}} / d t$ in the two problems. From (23), it is 
easy to see that the energy conservation in the homogenized problems reads

$$
\frac{d}{d t} \int_{\mathcal{D}_{h}} \mathrm{~d} V\left[\frac{\rho}{2} V^{\mathrm{h}^{2}}+\frac{1}{2 \mu}\left|\boldsymbol{\Sigma}^{\mathrm{h}}\right|^{2}\right]+\int_{X_{1}=0} \mathrm{~d} X_{2} V^{\mathrm{h}} \Sigma_{1}^{\mathrm{h}}+\int_{\mathcal{L}_{h}} \mathrm{~d} S \boldsymbol{\Pi} \cdot \mathbf{n}=0,
$$

where $\mathcal{L}_{h}=\partial \mathcal{D}_{h} \backslash\left(X_{1}=0\right)$ is the boundary of $\mathcal{D}_{h}$ except the segment at $X_{1}=0$. In view of the stability of numerical scheme, we want $I \equiv \int_{X_{1}=0} \mathrm{~d} X_{2} V^{\mathrm{h}} \Sigma_{1}^{\mathrm{h}} I \equiv \int_{X_{1}=0} \mathrm{~d} X_{2} V^{\mathrm{h}} \Sigma_{1}^{\mathrm{h}}$ to be the time derivative of a positive energy $\mathcal{E}_{b}$. Applying the boundary conditions of Eqs. (23), we get

$$
I=\int_{X_{1}=0} \mathrm{~d} X_{2}\left[(e \varphi-a) \frac{\partial \Sigma_{1}^{\mathrm{h}}}{\partial X_{1}} V^{\mathrm{h}}+h \mathcal{C} \frac{\partial \Sigma_{2}^{\mathrm{h}}}{\partial X_{2}} V^{\mathrm{h}}\right],
$$

leading to $I=\mathrm{d} \mathcal{E}_{b} / \mathrm{d} t$ (with (23) again), with

$$
\left\{\begin{array}{l}
\mathcal{E}_{b}=\frac{1}{2} \int_{X_{1}=0} \mathrm{~d} X_{2}\left[\rho(e \varphi-a) V^{\mathrm{h} 2}+h \mathcal{C}_{a} \frac{\Sigma_{2}^{2}}{\mu}\right], \\
h \mathcal{C}_{a} \equiv e \varphi-a-h \mathcal{C} .
\end{array}\right.
$$

Note that the integration by part of $V^{\mathrm{h}} \partial_{X_{2}} \Sigma_{2}^{\mathrm{h}}$ makes a boundary term to appear, $\left[\Sigma_{2}^{\mathrm{h}} V^{\mathrm{h}}\right]_{X_{2}}$ and something should be said at both extremities of the equivalent surface; this is disregarded in the present paper. To ensure $\mathcal{E}_{b} \geq 0$, one has to choose $a \leq e \varphi$ and to ensure $\mathcal{C}_{a} \geq 0$. In fact, this latter constraint is more restrictive since $\mathcal{C} \geq 0$. Indeed, coming back to the problem (17) satisfied by $W$, we have

$$
\begin{aligned}
0 & =\int_{\Omega} \mathrm{d} \mathbf{y} W \Delta W=\int_{\Omega} \mathrm{d} \mathbf{y} W \operatorname{div}\left(\boldsymbol{\nabla} W+\mathbf{e}_{2}\right) \\
& =-\int_{\Omega} \mathrm{d} \mathbf{y}\left(\boldsymbol{\nabla} W+\mathbf{e}_{2}\right) \boldsymbol{\nabla} W+\int_{\partial \Omega} \mathrm{d} l \boldsymbol{\nabla}\left(W+y_{2}\right) \cdot \mathbf{n} W,
\end{aligned}
$$

and the integral on $\partial \Omega$ vanishes because of the boundary conditions in (17). It results that

$$
\mathcal{C}=\int_{\Omega} \mathrm{d} \mathbf{y}|\boldsymbol{\nabla} W|^{2} \geq 0
$$

In the following, we inspect the bound on a to ensure that $h \mathcal{C} \leq e \varphi-a$ which guaranties $\mathcal{E}_{b} \geq 0$ in (24).

\subsubsection{Bounds for the a value ensuring a positive boundary energy $\mathcal{E}_{b}$}

To find a bound for $\mathcal{C}$ (and thus on a), it is sufficient to remark that the homogenized problem (17) admits a variational formulation from which a principle of energy minimization can be associated, specifically

$$
E(\mathbf{S}) \leq E(\tilde{\mathbf{S}}), \quad \text { with } E(\tilde{\mathbf{S}}) \equiv \int_{\Omega}\left|\tilde{\mathbf{S}}-\mathbf{e}_{2}\right|^{2},
$$

for any $\tilde{\mathbf{S}}$ admissible field satisfying $\tilde{\mathbf{S}} . \mathbf{n}_{\mid \Gamma}=0$ and $\tilde{\mathbf{S}} \rightarrow \mathbf{e}_{2}$ when $y_{1} \rightarrow+\infty$, and

$$
E(\mathbf{S})=\int_{\Omega}|\nabla W|^{2}, \quad \text { with } \mathbf{S}=\boldsymbol{\nabla}\left(W+y_{2}\right) .
$$


Using (26), we must have

$$
\mathcal{C}=E(\mathbf{S}) \leq E(\tilde{\mathbf{S}})
$$

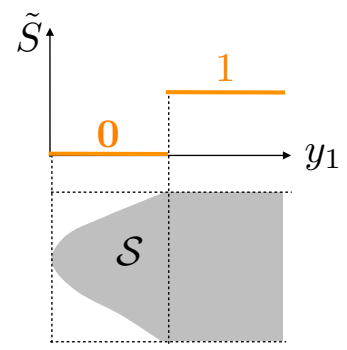

Fig. 4. The chosen admissible field $\tilde{\mathbf{S}} ; \tilde{\mathbf{S}}=\mathbf{0}$ in the roughness and $\tilde{\mathbf{S}}=\mathbf{e}_{2}$ outside

We choose $\tilde{\mathbf{S}}$ piecewise constant along $y_{1}$ : for the $y_{1}$ values inside the roughness, $\tilde{\mathbf{S}}=\mathbf{0}$ and outside $\tilde{\mathbf{S}}=\mathbf{e}_{2}$ (Fig. 4). For this field, we have

$$
\tilde{\mathbf{S}}=\mathbf{e}_{2}
$$

from which $\mathcal{C} \leq \mathcal{S}$ imposes the final (sufficient but not necessary) condition

$$
\mathcal{E}_{b} \geq 0, \quad \text { if } a \leq 0,
$$

and this holds for any shape of the roughnesses. Obviously, a less strict criterion can be found for particular a shape. Fig. 5 reports the variation of $\mathcal{C}_{a}$ varying $a$ and $\varphi$ for rectangular roughness shape as we shall consider in the forthcoming section. It is visible that increasing $\varphi$ produce larger values of $a$ allowing for $\mathcal{C}_{a} \geq 0$; from the estimate $\mathcal{C} \simeq$ $\varphi e / h-\pi \varphi^{2} / 16$ given in [5], we get $\mathcal{C}_{a} \simeq-a / h+\pi \varphi^{2} / 16$, and the line $a / h=\pi \varphi^{2} / 16$ reasonably corresponds to $\mathcal{C}_{a}=0$ (dotted black line in Fig. 5).

\subsection{Accuracy of the homogenized solution with respect to the actual solution}

In this section, we inspect the accuracy of the homogenization and to do so, we shall consider the particular scattering problem of the reflection of a wave hitting the roughnesses at oblique incidence $\theta$. We shall work with complex fields (owing to the physical fields are the real parts of the computed complex ones); in the harmonic regime, the complex fields (and we shall consider the displacement field $U(\mathbf{X})$ ) have a time dependance in $e^{-\mathrm{i} k c t}$ and it will be omitted in the following. For an incident wave of the form

$$
U^{\mathrm{inc}}(\mathbf{X})=e^{-\mathrm{i} k \cos \theta X_{1}+\mathrm{i} k \sin \theta X_{2}},
$$

we discriminate the right-going wave, with $X_{1}$ dependence in $e^{i k \cos \theta X_{1}}$ (corresponding to increasing $X_{1}$ values for increasing time) and left-going waves with $X_{1}$-dependence in $e^{-i k \cos \theta X_{1}}$ (the incident wave is a left-going wave coming from $X_{1} \rightarrow+\infty$ ). In the actual problem, this is done in the configuration of Fig. 6(a). The wavefield for $X_{1}>0$ reads

$$
U(\mathbf{X})=\left[e^{-\mathrm{i} k \cos \theta X_{1}}+R e^{\mathrm{i} k \cos \theta X_{1}}\right] e^{\mathrm{i} k \sin \theta X_{2}}+U^{\mathrm{ev}}(\mathbf{X}) .
$$




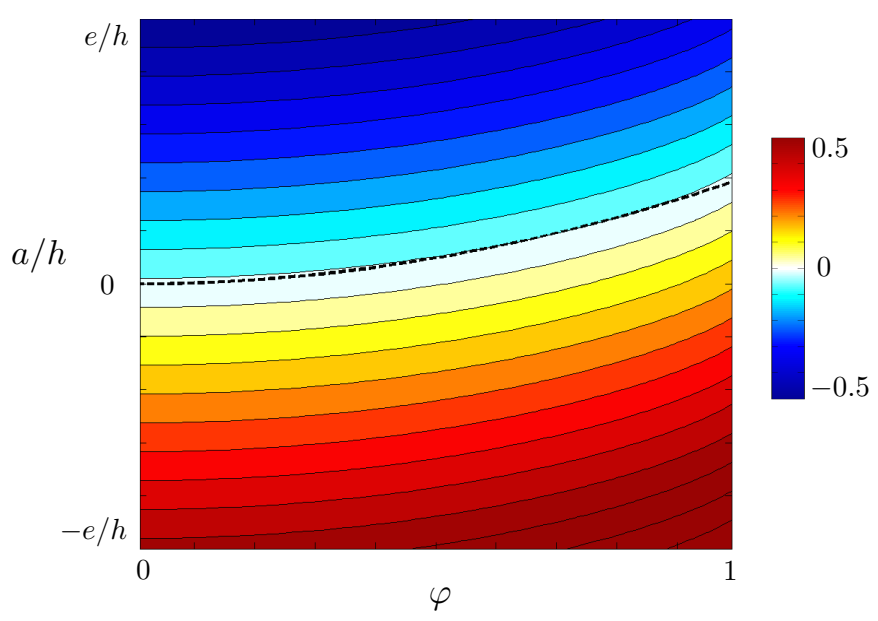

Fig. 5. $\mathcal{C}_{a}$ in (24) (with (21)) in colorscale as a function of $a / h$ and $\varphi$ for rectangular shape roughness (and $e / h=0.5)$; the dotted black line shows $a / h=\pi \varphi^{2} / 16$ where $\mathcal{C}_{a} \simeq 0$

The boundary condition on the roughnesses are Neumann boundary condition $\boldsymbol{\nabla} U . \mathbf{n}=0$, with $\mathbf{n}$ the local normal to the roughnesses. $U^{\mathrm{ev}}(\mathbf{X})$ is an evanescent field excited in the vicinity of the roughnesses and vanishing at $X_{1} \rightarrow+\infty$. It is worth noting that $U^{\mathrm{ev}}(\mathbf{X})=0$ for $\varphi=0$ and $\varphi=1$, since the problem is reduced to a one-dimensional problem along $X_{1}$. With $\mathbf{n}=\mathbf{e}_{1}$ in these cases, the boundary conditions are $\partial_{X_{1}} U\left(0, X_{2}\right)=0$ $(\varphi=0)$ and $\partial_{X_{1}} U\left(-e, X_{2}\right)=0(\varphi=1)$; it is easy to see that exact solutions are $R=1$ and $R=e^{2 \mathrm{ike} \cos \theta}$ respectively (and $U^{\mathrm{ev}}(\mathbf{X})=0$ in both cases).

The reflection coefficient, with $-|R|=1$ by conservation of the energy, is characterized by its phase. As written in (32), the phase of the reflection is defined by

$$
R=\frac{U_{\mid \Sigma}^{r}}{U_{\mid \Sigma}^{\text {inc }}},
$$

with $U^{\mathrm{r}}=\left(U-U^{\mathrm{inc}}\right)$ and where we choose the plane $\Sigma$ corresponding to boundary between the roughnesses and the plain elastic body, Fig. 6.

The homogenized problem (23) can be translated in terms of a complex displacement field $U^{\mathrm{h}}$. With $V^{\mathrm{h}} \rightarrow-\mathrm{i} \omega U^{\mathrm{h}}$, we get $\Sigma_{i}^{\mathrm{h}} \rightarrow \mu \partial_{X_{i}} U^{\mathrm{h}}$, where the arrow means going toward complex fields. (23) can be written in terms of the complex field of displacement $U^{\mathrm{h}}$ only, namely

$$
\left\{\begin{array}{l}
\Delta U^{\mathrm{h}}+k^{2} U^{\mathrm{h}}=0, \quad \text { for } X_{1}>0, \\
\frac{\partial U^{\mathrm{h}}}{\partial X_{1}}\left(0, X_{2}\right)=(e \varphi-a) \frac{\partial^{2} U^{\mathrm{h}}}{\partial X_{1}^{2}}\left(0, X_{2}\right)+h \mathcal{C} \frac{\partial^{2} U^{\mathrm{b}}}{\partial X_{2}^{2}}\left(0, X_{2}\right), \\
\lim _{X_{1} \rightarrow+\infty}\left[\frac{\partial U^{\mathrm{h}, \mathrm{r}}}{\partial X_{1}}-\mathrm{i} k \cos \theta U^{\mathrm{h}, \mathrm{r}} \rightarrow 0\right], \quad \text { with } U^{\mathrm{h}, \mathrm{r}} \equiv U^{\mathrm{h}}-U^{\mathrm{inc}} .
\end{array}\right.
$$




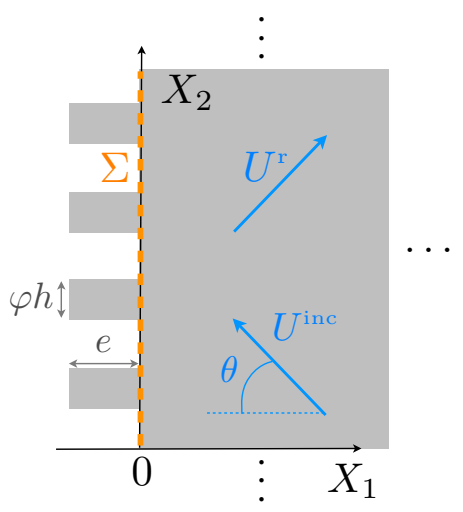

(a)

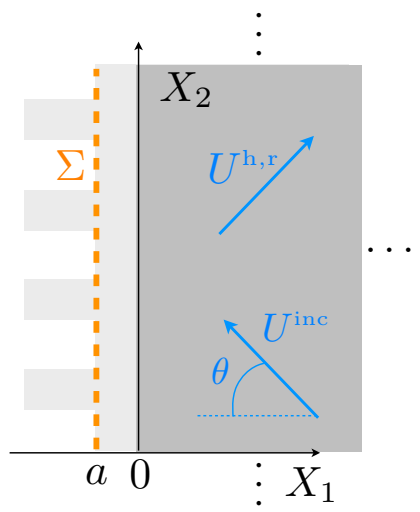

(b)

Fig. 6. Reflection of a wave $U^{\text {inc }}$ at oblique incidence on the rough boundary of the elastic body; (a) in the real problem with $U^{\mathrm{r}}$ the reflected wave and (b) in the homogenized problem with $U^{\mathrm{h}, \mathrm{r}}$ the reflected wave (the system of coordinate has been shifted of $a$, here $a<0$; light grey region show the actual roughnesses, but they do not exist in the homogenized problem)

In the above system, the origine of the $X_{1}$-axis has been shifted of $a$ (Fig. 6); to keep the same definition of the reflection coefficient, we want

$$
R^{\mathrm{h}}=\frac{U_{\mid \Sigma}^{\mathrm{h}, r}}{U_{\mid \Sigma}^{\mathrm{hic}},}
$$

and now $\Sigma$ is the plane $X_{1}=a$. An exact solution of (23) can be found, accounting for (35), of the form

$$
U^{\mathrm{h}}(\mathbf{X})=\left[e^{-\mathrm{i} k \cos \theta\left(X_{1}-a\right)}+R^{\mathrm{h}} e^{\mathrm{i} k \cos \theta\left(X_{1}-a\right)}\right] e^{\mathrm{i} k \sin \theta \mathrm{X}_{2}},
$$

and applying the boundary condition of (23) at $X_{1}=0$, we have

$$
R^{\mathrm{h}}=\frac{1+\mathrm{i} k h \cos \theta z_{a}(\theta)}{1-\mathrm{i} k h \cos \theta z_{a}(\theta)} e^{2 \mathrm{i} k a \cos \theta}, \quad z_{a}(\theta) \equiv\left(\varphi \frac{e}{h}-\frac{a}{h}\right)+\mathcal{C} \tan ^{2} \theta .
$$

To begin with, we report in Fig. 7 an example of the wavefield in the actual problem together with its homogenized counterparts obtained for $a=-e$ and $a=0$ (the configuration is described in the figure caption). The agreement $\left|U-U^{\mathrm{h}}\right| /|U|$ (in $\mathrm{L}_{2}$ norm) is of $20 \%$ for $a=-e$ and of $5 \%$ for $a=0$, which suggests that small $a$ value is preferable.

In fact, something is not very convenient in the form of (37). We have found that at leading order, the roughnesses behave as a flat free boundary, and as previously said, this will be exact in the two limiting cases $\varphi=0$ (leading to $R=1$ ) and $\varphi=1$ (leading to $R=$ $\left.e^{2 \mathrm{i} k e \cos \theta}\right)$. In both cases, the elementary problem (17) has a trivial solution, $W=$ constant, since $\Gamma$ is a boundary $y_{1}=$ constant, whence $\mathcal{C}=0$. But neither $e$ nor $a$ being zero, $R^{\mathrm{h}}$ does not go to the expected values; strictly, this is possible since the homogenized solution is an approximation of the real one, but it is annoying to constat that going to higher order 
may degrade the prediction, even if this concerns limiting cases only. To avoid this, it is possible to renormalized the reflection coefficient (37) identifying $(1+\mathrm{i} a \varepsilon) /(1-\mathrm{i} a \varepsilon)$ to $e^{2 i a \varepsilon}$ which is true up to $O\left(\varepsilon^{2}\right)$. We get

$$
R^{\text {ren }}=e^{\mathrm{i} \psi}, \quad \psi \equiv 2 k \cos \theta\left(\varphi e+\mathcal{C} \tan ^{2} \theta\right),
$$

and this renormalized version goes to the expected values for $\varphi=0,1$.

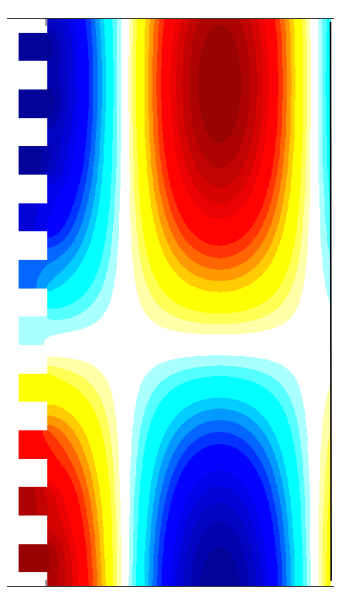

(a) $U(\mathbf{X})$

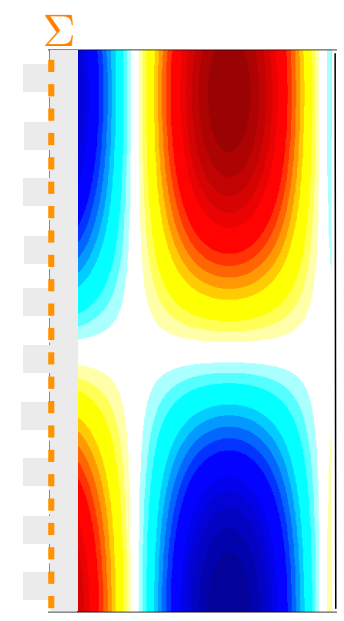

(b) $U^{\mathrm{h}}(\mathbf{X}), a=-e$

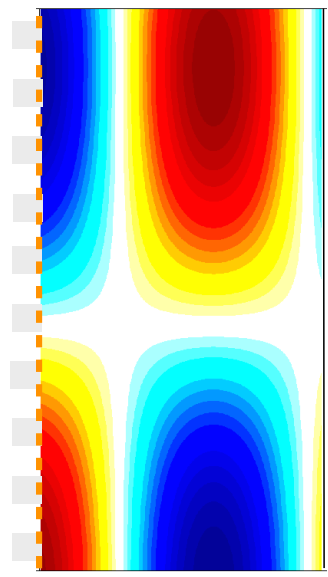

(c) $U^{\mathrm{h}}(\mathbf{X}), a=0$

Fig. 7. Reflection of an incident wave on the free edge of an elastic body with step roughnesses $\left(k h=1, e / h=\varphi=0.5, \theta=20^{\circ}\right)$. (a) the solution in the actual problem $\mathrm{U}(\mathrm{X})$, (b-c) the homogenized solutions $U^{\mathrm{h}}(\mathrm{X}),(36)-(37)$, for $a=-e$ and $a=0$

We report in Fig. 8 two series of results. The color scale panels show $\left|R^{\mathrm{h}}-R\right|$ as a function of $\varphi$ for a varying between $-e$ and $e$. The error decreases when a increases, which is a bad news since a has an upper bound $(a \leq 0)$ in order to ensure a positive energy supported by the effective boundary $\left(\mathcal{E}_{b}>0\right)$, as discussed in the previous section. Next, the error increases when $\varphi$ or $\theta$ increases and in both cases, this is because $R$ departs from the value $R=1$ obtained in the absence of roughnesses, see e.g. (38). Next, the upper panels reports $\left|R^{\text {ren }}-R\right|$ (black lines) as a function of $\varphi$ together with the set of errors $\left|R^{\mathrm{h}}-R\right|$ corresponding to $a<0$. As expected, the error vanishes for $\varphi \rightarrow 0$; this is less true for $\varphi=1$ except for $\theta$ which does not question $\mathcal{C}$ (when $\mathcal{C}$ is concerned, only the singular value $\varphi=1$ would lead to $R^{\text {ren }}=R$ for $\mathcal{C}=0$; but until $\varphi=1, C$ increases). From (a) and (b) , we observe that Rren is a better estimation of $R$ and we built it indeed to do a better job; unexpectedly, it happens that this is not the case for high $\varphi$ values near the grazing angles and we do not have explanation for that. All the observations reported in Fig. 8 are recovered for all $k$ and $e$ values; increasing $k$ or $e$ only produce a global increase of the errors. 

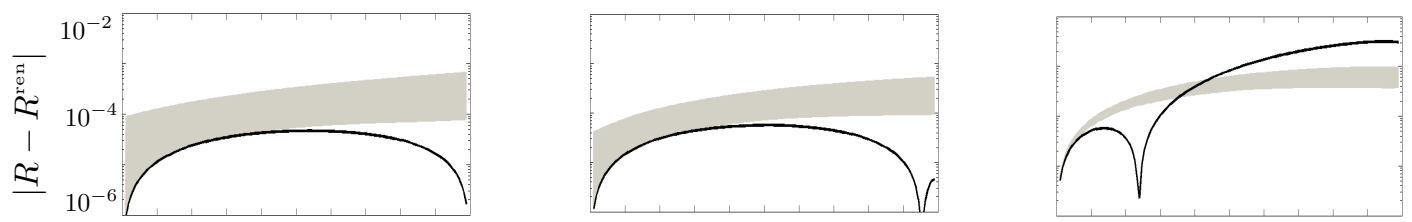

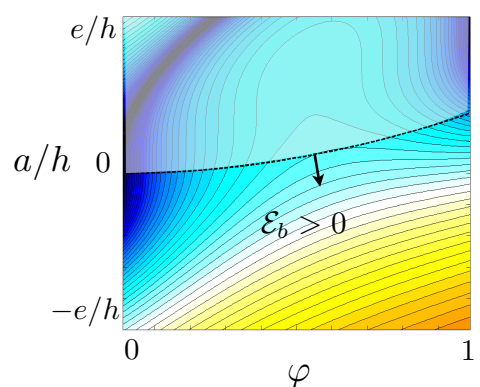

(a) $\theta=0$

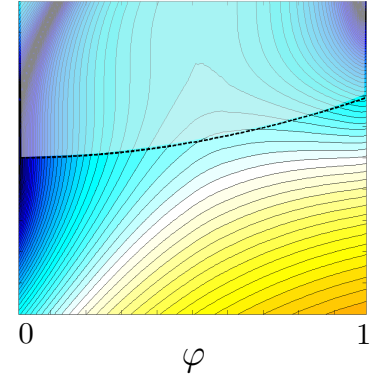

(b) $\theta=40^{\circ}$

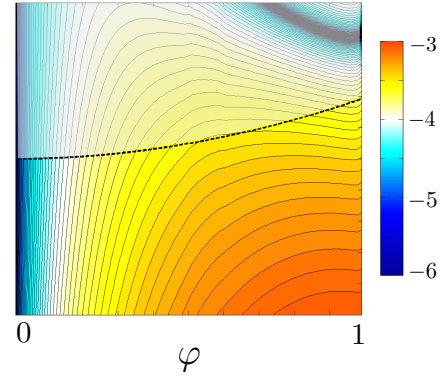

(c) $\theta=80^{\circ}$

Fig. 8. Top panels report the error $\left|R-R^{\text {ren }}\right|$ as a function of $\varphi$ (black lines) and the set of the errors $\left|R-R^{\mathrm{h}}\right|$ for $0<a<e$ (grey zones). Bottom panels report the error $|R-| R^{\mathrm{h}} \mid$ (in colorscale) in the plane $(\varphi, a / h)$; the dotted black line indicates the limit value of $a / h$ below which $\mathcal{E}_{b}>0$

\section{CONCLUDING REMARKS}

We have studied a homogenized problem which can replace the actual problem of the reflection of shear waves at the rough free boundary of an elastic body. Parameters characteristic of an equivalent flat boundary enter in a boundary condition which differs from the usual stress free condition. We have inspected different forms of the boundary conditions, being all equivalent up to the order of validity of the model. This has been done addressing two aspects, (i) wether or not the homogenized problem is well suited for a numerical resolution in the time domain (which means free of numerical instabilities in the time computation) and (ii) which formulation gives the smallest error in the model when compared to the solution of the actual problem. The first aspect questions the equation of energy conservation; the homogenized boundary condition makes an additional term of energy to appear and this energy must be positive in order ensure a consistent computational method in the time domain (and as so, it is not optional). The second aspect is of less importance; firstly because no definitive answer can be given, the error being measured in a particular scattering problem with no guaranty that the optimal homogenized problem will be the same in another scattering problem.

\section{REFERENCES}

[1] D. Cioranescu and P. Donato. An introduction to homogenization, Vol. 4. The Clarendon Press Oxford University Press, New York, (1999). 
Agnès Maurel, Jean-Jacques Marigo, Kim Pham

[2] J. J. Marigo and C. Pideri. The effective behavior of elastic bodies containing microcracks or microholes localized on a surface. International Journal of Damage Mechanics, 20, (8), (2011), pp. 1151-1177. https://doi.org/10.1177/1056789511406914.

[3] M. David, J. J. Marigo, and C. Pideri. Homogenized interface model describing inhomogeneities located on a surface. Journal of Elasticity, 109, (2), (2012), pp. 153-187. https://doi.org/10.1007/s10659-012-9374-5.

[4] A. Bonnet-Bendhia, D. Drissi, and N. Gmati. Simulation of muffler's transmission losses by a homogenized finite element method. Journal of Computational Acoustics, 12, (03), (2004), pp. 447-474. https://doi.org/10.1142/s0218396x04002304.

[5] J. J. Marigo and A. Maurel. Homogenization models for thin rigid structured surfaces and films. The Journal of the Acoustical Society of America, 140, (1), (2016), pp. 260-273. https://doi.org/10.1121/1.4954756.

[6] B. Delourme, H. Haddar, and P. Joly. Approximate models for wave propagation across thin periodic interfaces. Journal de Mathématiques Pures et Appliquées, 98, (1), (2012), pp. 28-71. https://doi.org/10.1016/j.matpur.2012.01.003.

[7] B. Delourme. High-order asymptotics for the electromagnetic scattering by thin periodic layers. Mathematical Methods in the Applied Sciences, 38, (5), (2015), pp. 811-833. https://doi.org/10.1002/mma.3110.

[8] A. Maurel, J. J. Marigo, and A. Ourir. Homogenization of ultrathin metallo-dielectric structures leading to transmission conditions at an equivalent interface. The Journal of the Optical Society of America B, 33, (5), (2016), pp. 947-956. https://doi.org/10.1364/josab.33.000947.

[9] J. J. Marigo and A. Maurel. Two-scale homogenization to determine effective parameters of thin metallic-structured films. Proc. R. Soc. A, 472, (2016).

[10] L. Rayleigh. On the dynamical theory of gratings. In Proceedings of the Royal Society of London, Series A, Vol. 79, (1907), pp. 399-416.

[11] P. A. Martin and R. A. Dalrymple. Scattering of long waves by cylindrical obstacles and gratings using matched asymptotic expansions. Journal of Fluid Mechanics, 188, (1988), pp. 465490. https://doi.org/10.1017/s0022112088000801.

\section{APPENDIX 1. SIMPLE PROCEDURE TO GET $\mathcal{C}$ FOR RECTANGULAR INCLUSIONS}

Mode matching is a simple way to get $\mathcal{C}$. We consider the solution $\tilde{W}=W+y_{2}$ satisfying $\Delta \tilde{W}=0, \nabla \tilde{W} \cdot \mathbf{n}_{\mid \partial \vee}=0$ and $\tilde{W} \rightarrow y_{2}$ for $y_{1} \rightarrow \infty$. The field $\tilde{W}$ can be written

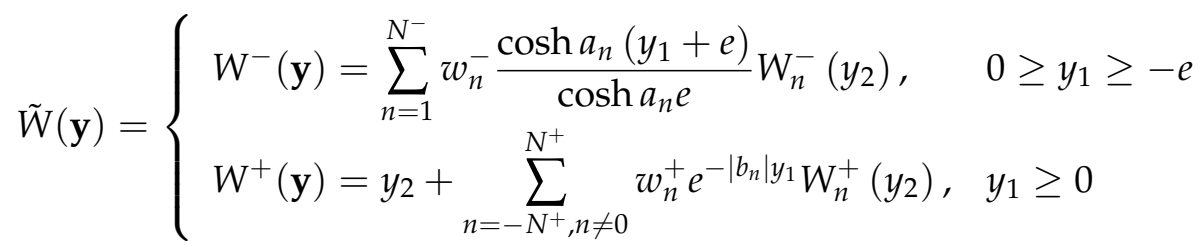

with $a_{n}=n \pi / \varphi, b_{n}=2 n \pi$, and

$$
W_{n}^{-}\left(y_{2}\right)=\sqrt{\frac{2}{\varphi}} \cos \left(a_{n} y_{2}+\frac{n \pi}{2}\right), \quad W_{n}^{+}\left(y_{2}\right)=e^{\mathrm{i} b_{n} y_{2}},
$$

the transverse functions (forming a basis) adapted for solutions being respectively periodic and with zero derivatives at $y_{2}= \pm \varphi / 2$ (Fig. 9). 


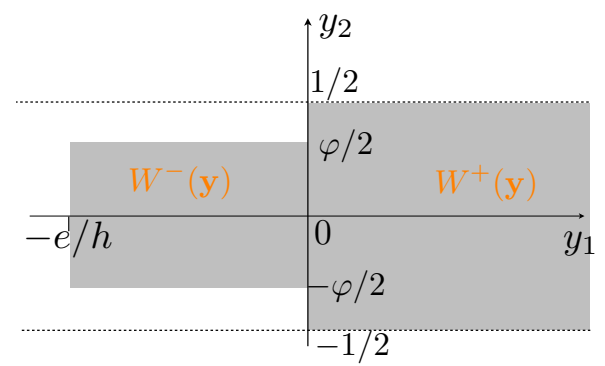

Fig. 9. Mode matching configuration. The solution $W^{ \pm}$is written for $y_{1}>-e / h,(39)$, and the resolution involves only matching conditions at $y_{1}=0$

Now, we will ask to $W^{ \pm}$to match (on average) their value and their first derivative at $y_{1}=0$, and this latter matching on the derivative will be done accounting for the boundary conditions at $y_{1}=0$ and $\left|y_{2}\right|>\varphi / 2$ (note that $W^{-}$satisfies by construction the right boundary condition on $\Gamma$, both at the wall $y_{1}=0$ and on the boundary of the roughness at $y_{2}= \pm \varphi / 2$ ). To that aim, we use the following relations

$$
\left\{\begin{array}{l}
\int_{-\varphi / 2}^{\varphi / 2} \mathrm{~d} y_{2} W^{-}\left(0, y_{2}\right) W_{m}^{-}\left(y_{2}\right)=\int_{-\varphi / 2}^{\varphi / 2} \mathrm{~d} y_{2} W^{+}\left(0, y_{2}\right) W_{m}^{-}\left(y_{2}\right), \\
\int_{-\varphi / 2}^{\varphi / 2} \mathrm{~d} y_{2} \frac{\partial W^{-}}{\partial y_{1}}\left(0, y_{2}\right) W_{m}^{+*}\left(y_{2}\right)=\int_{-1 / 2}^{1 / 2} \mathrm{~d} y_{2} \frac{\partial W^{+}}{\partial y_{1}}\left(0, y_{2}\right) W_{m}^{+*}\left(y_{2}\right),
\end{array}\right.
$$

with $W_{m}^{+*}$ the conjugate of $W_{m}^{+}\left(W_{m}^{-}\right.$is real). The first relation is the matching of the values in the region where $W_{m}^{-}$is defined. The second relation has more information: we have used that the $\partial_{y_{1}} W^{+}=0$ for $\left|y_{2}\right|>\varphi / 2$, from which

$$
\int_{-1 / 2}^{1 / 2} \mathrm{~d} y_{2} \frac{\partial W^{+}}{\partial y_{1}}\left(0, y_{2}\right) W_{n}^{+*}\left(y_{2}\right)=\int_{-\varphi / 2}^{\varphi / 2} \mathrm{~d} y_{2} \frac{\partial W^{+}}{\partial y_{1}}\left(0, y_{2}\right) W_{m}^{+^{*}}\left(y_{2}\right)
$$

afterwards we ask, on average, $\partial_{y_{1}} W^{+}=\partial_{y_{1}} W^{-}$for $\left|y_{2}\right|<\varphi / 2$. We get for a matrix system for the two vectors $\mathbf{w}^{-}=\left(w_{n}^{-}\right)_{n=0, \ldots, N-}$ and $\mathbf{w}^{+}=\left(w_{n}^{+}\right)_{n=0, \ldots, N+}$

$$
\left(\begin{array}{cc}
\mathrm{I} & -{ }^{t} \mathrm{~F}^{*} \\
\mathrm{FA} \tanh (\mathrm{A} e) & \mathrm{B}
\end{array}\right)\left(\begin{array}{l}
\mathbf{w}^{-} \\
\mathbf{w}^{+}
\end{array}\right)=\left(\begin{array}{l}
\mathbf{S} \\
\mathbf{0}
\end{array}\right),
$$

with I the $N^{-} \times N^{-}$identity matrix, $\mathrm{A}=\operatorname{diag}\left(a_{n}\right), \mathrm{B}=\operatorname{diag}\left(\left|b_{n}\right|\right)$, and $F_{m n}=$ $\int_{-\varphi / 2}^{\varphi / 2} \mathrm{~d} y_{2} W_{m}^{+*}\left(y_{2}\right) W_{n}^{-}\left(y_{2}\right)$ and $S_{n}=\int_{-\varphi / 2}^{\varphi / 2} \mathrm{~d} y_{2} y_{2} W_{n}^{-}\left(y_{2}\right)$. The expressions of $F_{m n}$ and $S_{n}$ are given below

$$
\left\{\begin{array}{l}
F_{m n}=\sqrt{\frac{\varphi}{2}}\left[\operatorname{sinc}\left(\left(a_{n}-b_{m}\right) \varphi / 2\right) e^{\mathrm{i} n \pi / 2}+\operatorname{sinc}\left(\left(a_{n}+b_{m}\right) \varphi / 2\right) e^{-\mathrm{i} n \pi / 2}\right], \\
S_{n}=-2 \sqrt{\frac{2}{\varphi}} \frac{1}{a_{n}^{2}}
\end{array}\right.
$$


The system of the form $M \mathbf{w}=\mathbf{s}$ with the matrix $M$ being square (this is not always the case in systems written using mode matching). Next, $M$ is invertible and the system can be solved to find $\mathbf{w}$ in the least squares sense (as done by the operation $M \backslash \mathbf{s}$ in Matlab). Then, we want to determine

$$
\mathcal{C}=-\int \mathrm{dy} \frac{\partial W}{\partial y_{2}}=\int_{-e}^{0} \mathrm{~d} y_{1} \int_{-\varphi / 2}^{\varphi / 2}\left[1-\frac{\partial W^{-}}{\partial y_{2}}\right] .
$$

where we have used that $W\left(y_{1} \geq 0, y_{2}\right)=W^{+}(\mathbf{y})-y_{2}$ is periodic, thus of vanishing contribution. It is now sufficient to write $\mathcal{C}=e \varphi-w_{n}^{-} \tanh a_{n} e / a_{n}\left[W_{n}^{-}\right]_{-\varphi / 2}^{\varphi / 2}$ to get

$$
\mathcal{C}=e \varphi+2 \sqrt{\frac{2}{\varphi}} \sum_{n} \frac{\tanh a_{n} e}{a_{n}} w_{n}^{-} .
$$

The procedure of mode matching is longer to explain than to encode; below is a script working with Matlab.

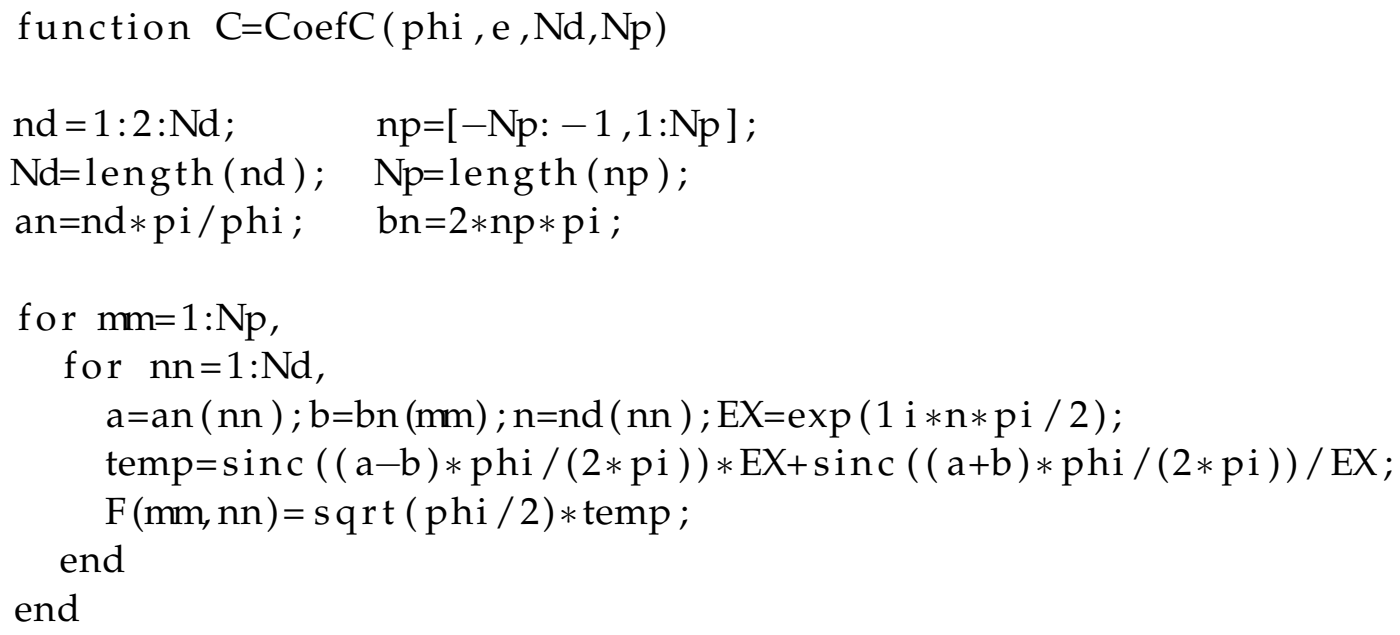

\section{APPENDIX 2. THE SAME PROCEDURE TO SOLVE THE SCATTERING PROBLEM}

The procedure to solve the scattering problem for an incident plane wave is quasi the same than to solve the static elementary problem. Now, we look for $U(\mathbf{X})$ being the 
solution of (32) (and the whole story is to compute $U^{\mathrm{ev}}(\mathbf{X})$ in $X_{1}>0$, which requires to compute $U\left(X_{1}<0, X_{2}\right)$ in the roughnesses).

$$
U(\mathbf{X})= \begin{cases}U^{-}(\mathbf{X})=\sum_{n=1}^{N^{-}} u_{n} \frac{\cos a_{n}\left(X_{1}+e\right)}{\cos a_{n} e} U_{n}^{-}\left(X_{2}\right), & 0 \geq X_{1} \geq-e \\ U^{+}(\mathbf{X})=e^{-\mathrm{i} k_{n} X_{1}} U_{0}^{+}\left(X_{2}\right)+\sum_{n=-N^{+}}^{N^{+}} R_{n} e^{i k_{n} X_{1}} U_{n}^{+}\left(y_{2}\right), & X_{1} \geq 0\end{cases}
$$

and with $\alpha_{n} \equiv n \pi / \varphi$ as previously, the bases are defined as

$$
U_{n}^{-}\left(X_{2}\right)=\sqrt{\frac{2-\delta_{n 0}}{\varphi}} \cos \left(\alpha_{n} X_{2}+\frac{n \pi}{2}\right), \quad a_{n}^{2}=k^{2}-\alpha_{n}^{2}
$$

which ensures that $U^{-}$satisfies the right Neumann boundary conditions for $X_{1}<0,\left|X_{2}\right|=$ $\varphi / 2$ and satisfies the Helmholtz equation $\Delta U^{-}+k^{2} U^{-}=0$, and

$$
U_{n}^{+}\left(X_{2}\right)=e^{i \beta_{n} X_{2}}, \quad \beta_{n}^{2} \equiv k^{2}-\left(k \sin \theta+\frac{2 n \pi}{h}\right)^{2} .
$$

Again, $U_{n}^{+}$is chosen in order to ensure (i) that $U^{+}$satisfies the Helmholtz equation $\Delta U^{+}+k^{2} U^{+}=0$ and (ii) that $U^{+}$satisfies the so-called condition of pseudo-periodicity $U^{+}\left(X_{1}, h\right)=U^{+}\left(X_{1}, 0\right) e^{i \beta_{0} h}$ (this condition is imposed by the form of the incident wave).

Now the same matching as (41) are used, leading to a matrix condition involving $G_{m n}=\int_{-\varphi / 2}^{\varphi / 2} \mathrm{~d} X_{2} U_{m}^{+*}\left(X_{2}\right) U_{n}^{-}\left(X_{2}\right)$,

$$
G_{m n}= \begin{cases}\sqrt{\varphi} \operatorname{sinc}\left(\beta_{m} \varphi / 2\right), & n=0 \\ \sqrt{\frac{\varphi}{2}}\left[\operatorname{sinc}\left(\left(\alpha_{n}-\beta_{m}\right) \varphi / 2\right) e^{\mathrm{i} n \pi / 2}+\operatorname{sinc}\left(\left(\alpha_{n}+\beta_{m}\right) \varphi / 2\right) e^{-\mathrm{i} n \pi / 2}\right], & n \neq 0\end{cases}
$$

We get a system of the form

$$
\left(\begin{array}{cc}
-{ }^{t} \mathrm{G}^{*} & \mathrm{I} \\
\mathrm{iK} & \mathrm{GA} \tan (\mathrm{A} e)
\end{array}\right)\left(\begin{array}{l}
\mathbf{R} \\
\mathbf{u}
\end{array}\right)=\left(\begin{array}{l}
\mathbf{s}_{1} \\
\mathbf{s}_{2}
\end{array}\right)
$$

The source terms are now due to the incident wave and we get

$$
S_{1, n}=G_{0 n}^{*}, \quad S_{2, n}=\mathrm{i} k_{0} \delta_{n 0} .
$$

The scripts below allow to compute the reflection coefficients $R_{n}$ (and $R=R_{0}$ ). For a graphical representation of the solution, see below.

function $[\mathrm{R}, \mathrm{Rn}, \mathrm{un}]=\mathrm{Scattering} \mathrm{Pb}(\mathrm{k}$, theta , e , phi , Np, Nn)

$\mathrm{np}=-\mathrm{Np}: \mathrm{Np} ; \mathrm{no}=\mathrm{Np}+1 ; \mathrm{nd}=0: \mathrm{Nn}$;

$\mathrm{Np}=$ length (np); Nd=length (nd);

beta $0=\mathrm{k} * \sin ($ theta $) ;$ betan $=$ beta $0+2 * n p * p i ；$ 


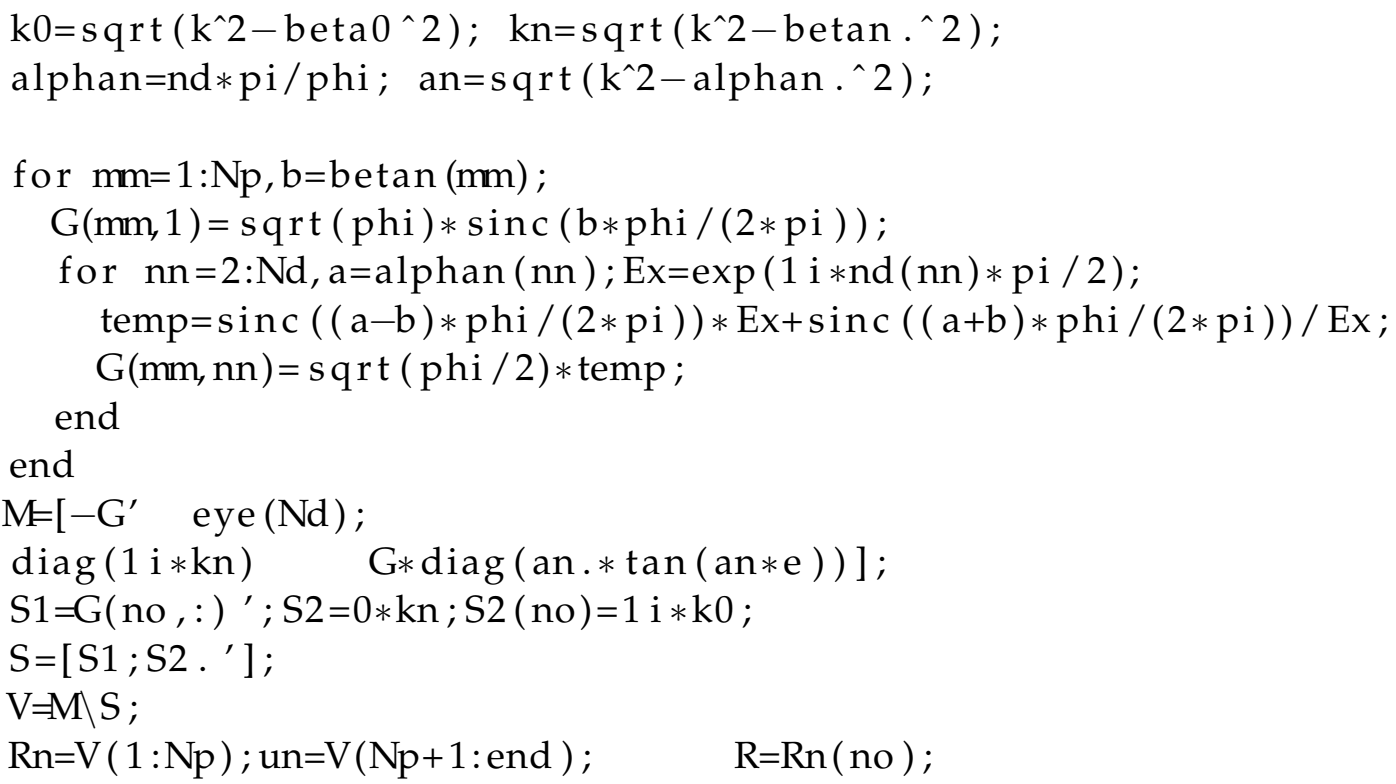

The graphical representation of the solution is done simply following (47). However, is $N^{-}$is too large, this may conduce to numerical divergences, because $a_{n}$ become complex, thus $\cos a_{n}\left(X_{1}+e\right) / \cos a_{n} e$ diverge for $X_{1}<0$. Obviously this limitation does not concerns the resolution of the system as presented above (since the mode matching is performed at $X_{1}=0$ and does not question $\left.X 1 ; 0\right)$. As given below, we simply use a trick to avoid divergence of the solution for $X_{1}<0$ ) (truncating the solution for diverging cos ane. Alternatively, one has to consider the solution written as

$$
U^{-}(\mathbf{X})=\sum_{n=1}^{N^{-}}\left[A_{n} e^{\mathrm{i} a_{n}\left(X_{1}+e\right)}+B_{n} e^{-\mathrm{i} a_{n} X_{1}}\right] U_{n}^{-}\left(X_{2}\right), \quad 0 \geq X_{1} \geq-e,
$$

and to apply matching conditions at $X_{1}=-e$ and $X_{1}=0$, and at $X_{1}=0$, it is

$$
\int_{-h \varphi / 2}^{h \varphi / 2} \mathrm{~d} X_{2} \frac{\partial U^{-}}{\partial X_{1}}\left(-e, X_{2}\right) U_{m}^{-}\left(X_{2}\right)=0,
$$

to get a final system on $\left(A_{n}, B_{n}, R_{n}\right)$.

function Graphic(k, theta,e,phi, Rn, un, L, Nrough,dx)

\%truncationifneeded,

alphan $=(0$ : length $($ un $)) *$ pi $/$ phi ; $\quad$ an $=\operatorname{sqrt}\left(k^{\wedge} 2-\right.$ alphan.$\left.^{\wedge} 2\right)$;

$[\max , \mathrm{Nd}]=\min (\operatorname{abs}(\cos (\mathrm{an} * \mathrm{e})-1 \mathrm{e} 10))$;

$\%$

$\mathrm{Np}=($ length $(\mathrm{Rn})-1) / 2$;

$\mathrm{np}=-\mathrm{Np}: \mathrm{Np} ; \quad \mathrm{nd}=0: \mathrm{Nd}-1$;

$\mathrm{Np}=$ length (np); Nd=length (nd); 


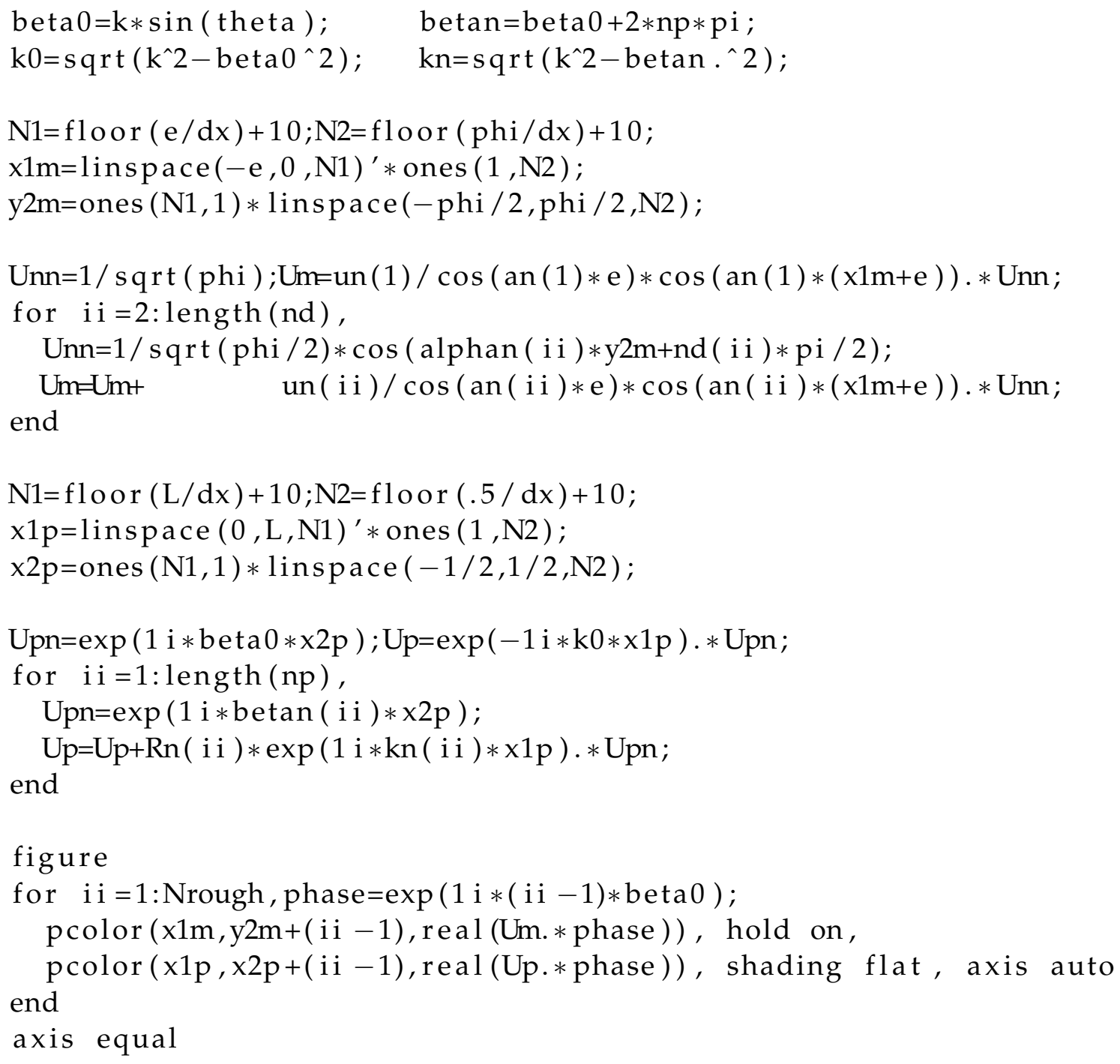

